\title{
Pulmonary arterial hypertension associated with protein kinase inhibitors: a pharmacovigilance-pharmacodynamic study
}

\author{
Lucie Cornet $^{1,12}$, Charles Khouri (10) $1,2,3,12$, Matthieu Roustit ${ }^{2,3}$, \\ Christophe Guignabert ${ }^{4,5,6}$, Marie-Camille Chaumais ${ }^{6,7,8}$, Marc Humbert $\mathbb{1}^{4,5,6}$, \\ Bruno Revol (10 ${ }^{1,3}$, Fabien Despas $9,10,11$, David Montani $\mathbb{1}^{4,5,6}$ and \\ Jean-Luc Cracowski ${ }^{1,2,3}$
}

Affiliations: ${ }^{1}$ Pharmacovigilance Unit, Grenoble Alpes University Hospital, Grenoble, France. ${ }^{2}$ Clinical Pharmacology Dept, INSERM CIC1406, Grenoble Alpes University Hospital, Grenoble, France. ${ }^{3}$ UMR $1042-$ HP2, INSERM, Université Grenoble Alpes, Grenoble, France. 'Université Paris-Sud, Faculté de Médecine, Université Paris-Saclay, Le Kremlin-Bicêtre, France. ${ }^{5}$ AP-HP, Service de Pneumologie, Hôpital Bicêtre, Le Kremlin-Bicêtre, France. 'INSERM UMR S 999, Hôpital Marie-Lannelongue, Le Plessis-Robinson, France. ${ }^{7}$ Université Paris-Sud, Faculté de Pharmacie Université Paris-Saclay, Châtenay Malabry, France. ${ }^{8}$ AP-HP Service de Pharmacie, Hôpital Bicêtre, Le Kremlin-Bicêtre, France. ${ }^{9}$ Medical and Clinical Pharmacology Unit, CHU Toulouse University Hospital, Toulouse, France. ${ }^{10}$ INSERM UMR1027, University of Toulouse III PaulSabatier, Toulouse, France. ${ }^{11}$ INSERM CIC 1436, Toulouse Clinical Investigation Centre, Toulouse, France.

${ }^{12}$ These two authors contributed equally to this work.

Correspondence: Charles Khouri, Unité de Pharmacologie Clinique, Centre d'Investigation Clinique de Grenoble, INSERM CIC1406, CHU Grenoble Alpes, 38043 Grenoble Cedex 09, France.

E-mail: ckhouridchu-grenoble.fr

@ERSpublications

Using the WHO pharmacovigilance database, PAH was found to be associated with dasatinib, bosutinib, ponatinib, ruxolitinib and nilotinib. The potential role of Src protein kinases and TEC in PAH induced by protein kinase inhibitors is further highlighted. http://ow.ly/56sO30nMKMj

Cite this article as: Cornet L, Khouri C, Roustit M, et al. Pulmonary arterial hypertension associated with protein kinase inhibitors: a pharmacovigilance-pharmacodynamic study. Eur Respir J 2019; 53: 1802472 [https://doi.org/10.1183/13993003.02472-2018].

ABSTRACT The pathophysiology of pulmonary arterial hypertension (PAH) induced by protein kinase inhibitors (PKIs) remains unclear. To gain knowledge into this rare and severe pathology we performed a study combining a pharmacovigilance approach and the pharmacodynamic properties of PKIs.

A disproportionality analysis on the World Health Organization pharmacovigilance database VigiBase using the reporting odds ratio (ROR) and 95\% confidence interval was first performed. Then, we identified the most relevant cellular targets of interest through a systematic literature review and correlated the pharmacovigilance signals with the affinity for the different PKIs. We further performed a hierarchical cluster analysis to assess patterns of binding affinity.

A positive disproportionality signal was found for dasatinib, bosutinib, ponatinib, ruxolitinib and nilotinib. Five non-receptor protein kinases significantly correlate with disproportionality signals: $c-S r c \quad(r=0.79$, $\mathrm{p}=0.00027)$, $\mathrm{c}$-Yes $(\mathrm{r}=0.82, \mathrm{p}=0.00015)$, Lck $(\mathrm{r}=0.81, \mathrm{p}=0.00046)$ and Lyn $(\mathrm{r}=0.80, \mathrm{p}=0.00036)$, all belonging to the Src protein kinase family, and TEC $(r=0.85, \mathrm{p}=0.00006)$. Kinases of the bone morphogenetic protein signalling pathway also seem to play a role in the pathophysiology of PKI-induced PAH. Interestingly, the dasatinib affinity profile seems to be different from that of other PKIs in the cluster analysis.

The study highlights the potential role of the Src protein kinase family and TEC in PAH induced by PKIs. This approach combining pharmacovigilance and pharmacodynamics data allowed us to generate some hypotheses about the pathophysiology of the disease; however, the results have to be confirmed by further studies. 


\section{Introduction}

Pulmonary hypertension is defined as an increase in mean pulmonary arterial pressure $\geqslant 25 \mathrm{mmHg}$ assessed by right heart catheterisation [1]. The pathophysiology is characterised by an increased migration and proliferation of pulmonary arterial smooth muscle cells, leading to vascular remodelling [2]. The classification proposed by the European Society of Cardiology/European Respiratory Society guidelines defines five groups of different pathological features which characterise the diverse clinical pulmonary hypertension groups [1]. Group 1 relates to pulmonary arterial hypertension (PAH), a rare and life-threatening condition characterised by the remodelling of pulmonary arteries [3], and associated with various aetiologies. Indeed, PAH may be idiopathic, heritable, drug and toxin induced, or associated with conditions such as connective tissue disease, HIV infection, congenital heart disease or schistosomiasis, with worldwide heterogeneity.

Among drug-induced $\mathrm{PAH}$, the multiple protein kinase inhibitor (PKI) dasatinib had been increasingly linked to PAH since $2009[4,5]$. More recently, several cases have reported potential association with or deterioration of pre-existing PAH with other PKIs such as bosutinib, ponatinib and lapatinib [6-8].

Since these compounds inhibit multiple kinases, the identification of a target responsible for such a rare adverse event is challenging. We thus mixed pharmacovigilance data mining with the pharmacodynamic properties of PKIs to gain knowledge into potential mechanisms underlying this rare and severe adverse event.

\section{Methods}

Study design

We first performed a disproportionality analysis from the World Health Organization (WHO) pharmacovigilance database VigiBase (www.who-umc.org/vigibase/vigibase). Disproportionality analyses are largely used by regulators to generate "pharmacovigilance signals" aiming at assessing putative links between drugs and adverse drug reactions (ADRs) [9]. Such methods compare the reporting proportion between a studied drug and all other drugs in the database for a given ADR. Several measures of disproportionality have been developed, but there is no recognised gold standard [10]. They do not provide risk quantification, but could be used as a proxy of the risk of an ADR when no other estimate is available (i.e. for extremely rare ADRs) [11-14]. In a second step we identified cellular targets of interest through a systematic literature review. Finally, we evaluated the association between the pharmacovigilance disproportionality signals and the affinity for different PKIs.

\section{Pharmacovigilance database}

VigiBase is the WHO global database of individual case safety reports (ICSRs). At the time of extraction, this database contained approximately 16 million reports of suspected adverse effects of medicines, from more than 150 countries, collected since 1968. VigiBase provides ICSRs with patient information such as sex, age, medical history and country; suspected and concomitant drugs taken with chronological information, as well as drug indication and dosage; and a description of the adverse effect with its severity and outcomes.

\section{Selection of cases}

We used the standardised high-level term "Pulmonary hypertensions" of MedDRA (Medical Dictionary for Regulatory Activities) terminology to identify pulmonary hypertension cases from VigiBase. To select drug-induced type $1 \mathrm{PAH}$ we excluded all ICSRs of pulmonary hypertension associated with cardiac, pulmonary or thrombotic disorders, connective tissue diseases, HIV infection, congenital heart disease, or schistosomiasis. Details are available in supplementary appendix S1.

Then, ICSRs containing drugs or toxins known to induce PAH (aminorex, fenfluramine, dexfenfluramine, benfluorex, amphetamines (dexamfetamine), phentermine and mazindol) were also excluded [15].

\section{Selection of PKIs}

To select PKIs with a reasonable level of information to calculate accurate reporting odds ratios (RORs), we included in the analysis only PKIs with more than 100 suspect ICSRs reported in VigiBase between January 1, 2002 and December 31, 2017 [16]. We therefore selected 28 drugs: afatinib, alectinib, axitinib, bosutinib, cabozantinib, ceritinib, cobimetinib, crizotinib, dabrafenib, dasatinib, erlotinib, gefitinib,

This article has supplementary material available from erj.ersjournals.com

Received: Dec 312018 | Accepted after revision: Feb 172019 
ibrutinib, lapatinib, lenvatinib, lestaurinib, osimertinib, nilotinib, palbociclib, pazopanib, ponatinib, regorafenib, ruxolitinib, sorafenib, sunitinib, trametinib, vandetanib and vemurafenib.

To avoid confounding in the pharmacovigilance signal interpretations, nintedanib and imatinib were excluded a priori from the selection because of suspected protopathic and indication bias. Indeed, it is impossible to distinguish reports of $\mathrm{PAH}$ induced by pulmonary fibrosis in nintedanib-treated patients and drug inefficacy in imatinib-treated patients from adverse events [17, 18].

Identification of protein kinases involved in PAH and affinity between PKIs and these targets Cellular targets of interest involved in PAH pathophysiology were identified through a systematic literature review in MEDLINE with the Medical Subject Headings ("Familial Primary Pulmonary Hypertension"[Mesh]) AND "Protein Kinases"[Mesh].

Affinity data for the targets of interest were extracted from the International Union of Basic and Clinical Pharmacology/British Pharmacological Society Guide to Pharmacology in 2018 [19].

\section{Disproportionality analysis}

We first performed a disproportionality analysis with the ROR method for each PKI of interest considered as suspect [20]. We compared the proportion of PAH reported for each PKI with the proportion of PAH associated with all other drugs used as non-cases. The cut-off for signal detection was defined as a ROR lower boundary $95 \%$ confidence interval $\geqslant 1$ and three or more cases [21]. We also performed a temporal analysis to assess to the influence of media safety alerts on the reporting rate of PAH among reported adverse events, as previously described [22].

\section{Statistical analyses}

To assess the link between the identified cellular targets of interest and pharmacovigilance signals, we calculated the Pearson correlation coefficient ( $r$ ) between the negative logarithm of the dissociation constant $K \mathrm{~d}(\mathrm{p} K \mathrm{~d})$ and the ROR.

We hypothesised that the higher the affinity for the cellular target, the higher the "risk" of notification of suspected drug-induced PAH. In order to take into account the multiplicity of comparisons, the statistical significance threshold for all p-values was adapted using a Bonferroni correction [23].

Sensitivity analyses were performed to assess the robustness of the results: 1) excluding PKIs that had less than three cases of PAH; 2) standardising the time on the market for the different PKIs at 6 years after the US Food and Drug Administration approval date, corresponding to the time between dasatinib approval and the first published safety alert; and 3) performing the correlation using other affinity data, extracted from Davis et al. [24].

We further performed a hierarchical cluster analysis, through the hierarchical $k$-means clustering method, to assess the similarity among receptor binding affinity profiles of the included protein kinases [25].

Lastly, for the PKIs associated with a significant pharmacovigilance disproportionality signal we studied the influence of media safety alerts on the reporting rate of PAH. Moreover, as suggested by a reviewer, we performed a multinomial regression analysis to assess the influence of dose and duration of exposure on the outcomes of the PAH cases (recovered/not recovered/died)

Descriptive results are expressed as mean with standard deviation or median (interquartile range (IQR)).

All analyses were performed using R version 3.2.3 (www.r-project.org).

\section{Results}

Selection of cases

Up to December 31, 2017, a total of 286834 ICSRs were related to the 22 selected PKIs. Among them, 733 cases of pulmonary hypertension were extracted. The exclusion of cases associated with other PAH aetiologies and concomitant drugs led to 442 ICSRs included in the final analysis (supplementary figure S1).

\section{Description of PAH cases}

Among the 442 cases of PAH, 193 were female (43.7\%), 202 were male (45.7\%) and sex was unknown for $47(10.6 \%)$; mean \pm SD age was $57.6 \pm 15.8$ years. A pleural effusion was associated with $\mathrm{PAH}$ in 75 cases (17.0\%). The median (IQR) delay between PAH and PKI introduction was 23 (6.3-41.3) months (data available for 206 ICSRs), with substantial heterogeneity: 2.9 (1.7-12.8) months for bosutinib, 27.9 (11.545.0) months for dasatinib, 11.7 (2.6-22.0) months for nilotinib, 10.7 (8.1-11.4) months for ponatinib and 12.0 (3.9-49.1) months for ruxolitinib. 
Identification of protein kinases involved in PAH

35 protein kinases involved in $\mathrm{PAH}$ pathophysiology were identified through the literature review (supplementary figure S2): ALK1/5, AMPKa1/2, BMPR1/2, B-Raf, c-Yes, DDR1, EIF2K4, ERBb1, FAK, FGFR1/2, HER2, IGF1R, JAK1/2, JNK1/2, KIT, Lck, Lyn, HGF, PDGFR $\alpha / \beta$, PKG, RAF1, ROCK2, Src, TEC, TIE2 and VEGFR1/2/3. Full definitions/aliases and the most relevant references about the target of interest are reported in supplementary appendix S2.

\section{Disproportionality analysis}

Among the 28 selected PKIs, at least one PAH case was reported for 22. A positive disproportionality signal was found for dasatinib, bosutinib, ponatinib, ruxolitinib and nilotinib, with a ROR of $28.64(95 \%$ CI 25.53-31.93), 13.43 (95\% CI 8.65-20.87), 3.88 (95\% CI 1.86-7.46), 3.71 (95\% CI 2.44-5.65) and 3.39 (95\% CI 2.43-4.73), respectively. RORs are represented in figure 1. Results of the sensitivity analysis (standardising on time on the market) were consistent with the main analysis, except for nilotinib which became nonsignificant. Results are presented in supplementary appendix S3 and supplementary figure S3.

Drug dosages were available for 295 cases and are represented in figure 2. Among the $170 \mathrm{PAH}$ cases associated with dasatinib, only two were reported with a dosage higher than recommended. No correlation was found between PKI dosage, duration of exposure and outcome severity (data not shown).

\section{Correlation analysis}

Among the 22 PKIs identified in VigiBase, affinity data for the target of interest were available for 16 [19]. Five protein kinases were significantly correlated with disproportionality signals: $c-S r c(r=0.79, p=0.00027)$, c-Yes $(r=0.82, p=0.00015)$, Lck $(r=0.81 \mathrm{p}=0.00046)$, Lyn $(\mathrm{r}=0.80, \mathrm{p}=0.00036)$ and TEC $(\mathrm{r}=0.85$, $\mathrm{p}=0.00006)$. The proportion of variance $\left(\mathrm{r}^{2}\right)$ explained by the model was $0.72,0.67,0.64,0.64$ and 0.72 for c-Src, c-Yes, Lck, Lyn and TEC, respectively. The results of the correlation analysis for each target classified according to its main cellular function are presented in figure 3.

Results for c-Yes, c-Src and TEC remained significant in all three sensitivity analyses, while results for Lck and Lyn remained significant in two of them. Furthermore, two other targets became significantly associated with disproportionality signals in the sensitivity analysis excluding PKI with less than three PAH cases: ALK1 $(r=0.9)$ and ALK5 $(r=0.98)$. Results are presented in supplementary appendix S4.

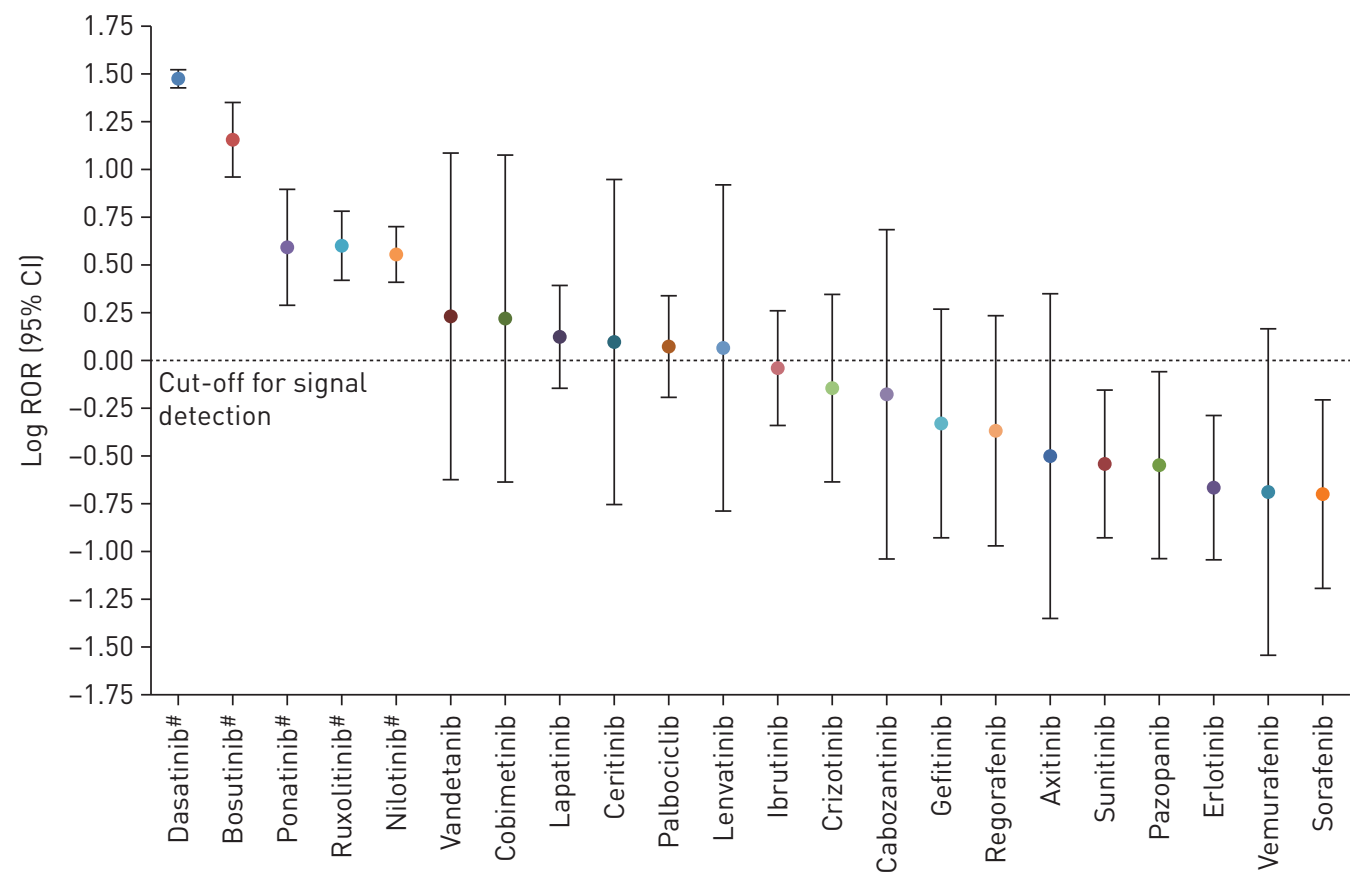

FIGURE 1 Forest plot of the reporting odds ratio (ROR) values of protein kinase inhibitor (PKI)-related pulmonary arterial hypertension. ${ }^{\#}$ : PKI associated with positive disproportionality signal. 


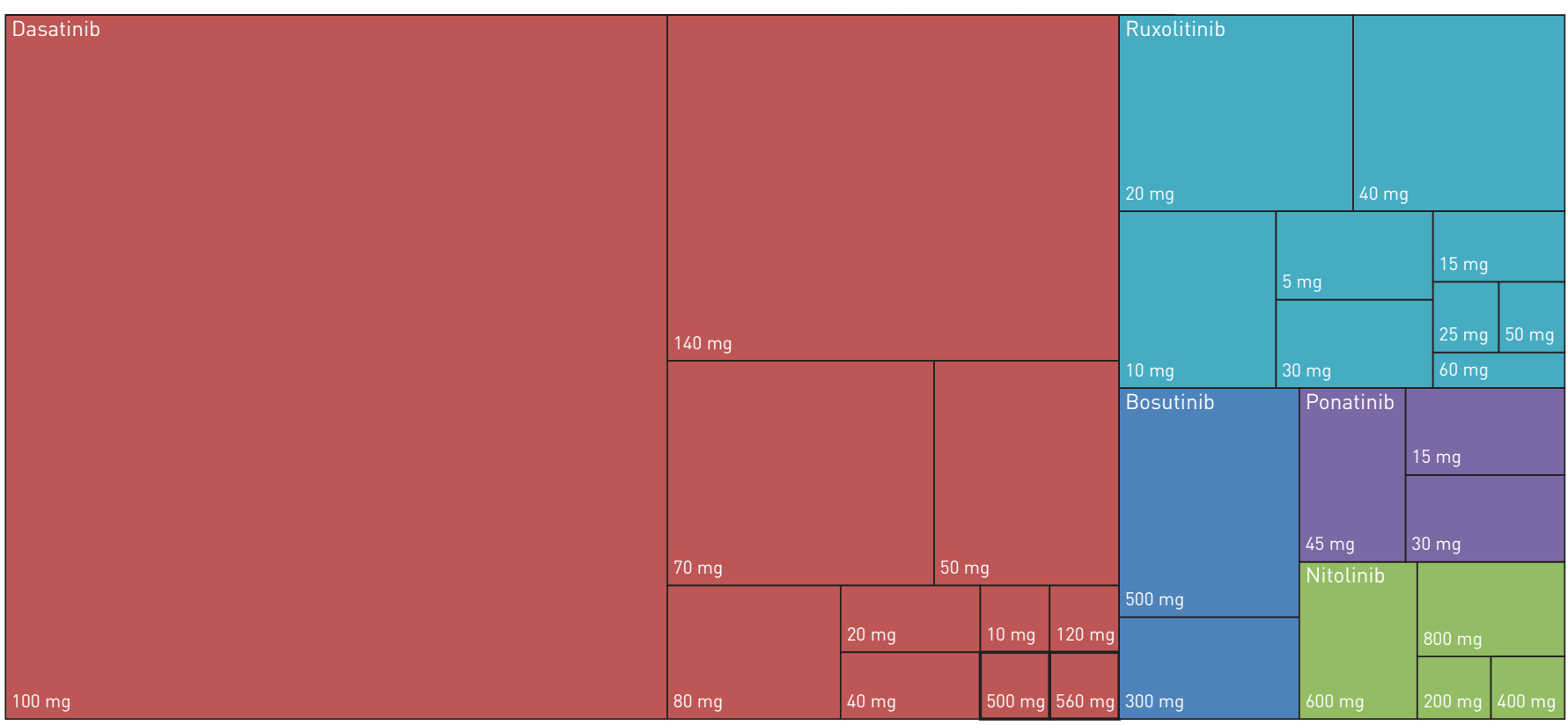

FIGURE 2 Treemap of daily drug dosages for the five most reported protein kinase inhibitors: dasatinib ( $n=170$ ), bosutinib ( $n=13$ ), ruxolitinib $(n=36)$, nilotinib $(n=9)$ and ponatinib $(n=10)$. The area of the rectangles is proportional to the number of reported cases for each dosage/drug combination. Higher than recommended dosages of dasatinib (500 and $560 \mathrm{mg}$ ) are indicated.

\section{Cluster analysis}

We performed a hierarchical clustering based on the affinity data of each PKI. Results are presented in figure 4, which represents the degree of PKI affinity for the identified protein kinase involved in PAH. The dasatinib affinity profile differs from that of bosutinib, ruxolitinib and nilotinib.

\section{Time-trend analysis}

We studied the association between PAH reports and media safety alerts by a temporal analysis of the annual proportion of PAH reports for 1000 reported adverse events for each PKI with a significant pharmacovigilance disproportionality signal. Notably, an important increase in the rate of notification for dasatinib and bosutinib can be seen after first media alert. Results are presented in figure 5.

\section{Discussion}

To the best of our knowledge, this is the first pharmacovigilance analysis assessing the reporting risk of $\mathrm{PAH}$ associated with PKI use. Among more than 16 million ADRs reported in the WHO

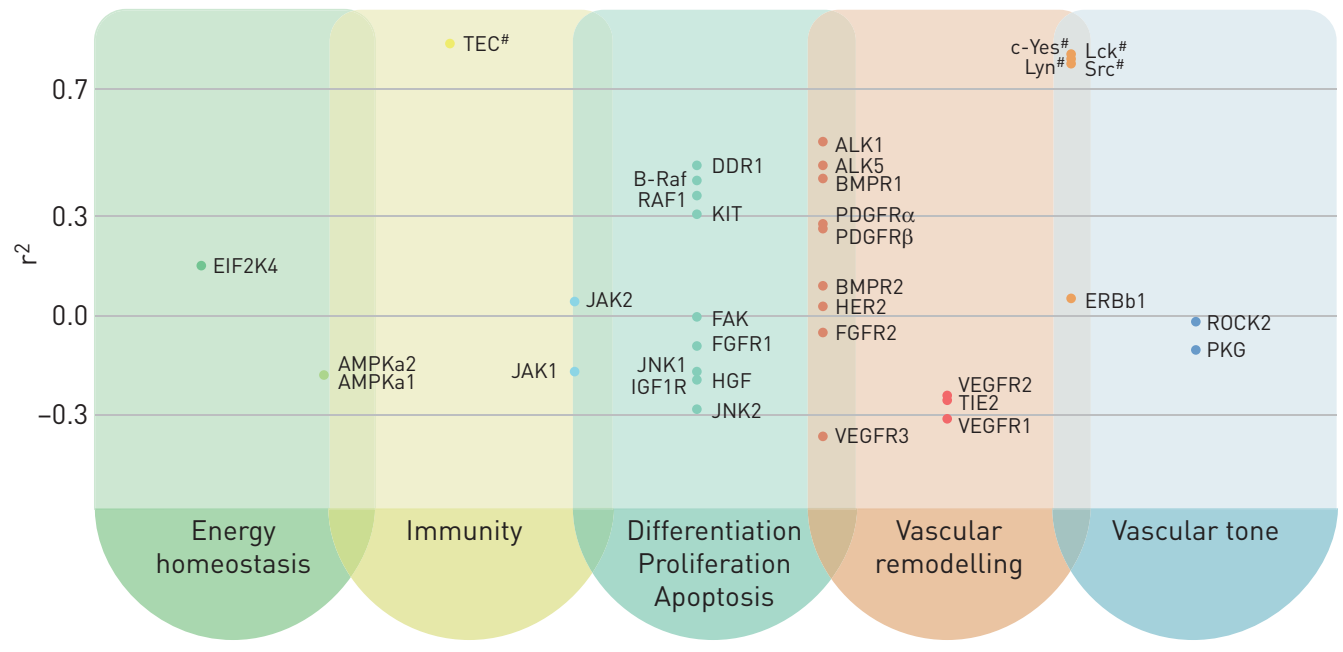

FIGURE 3 Manhattan plot synthesising the correlation analysis. Pearson correlation coefficients $\left(r^{2}\right)$ of each target classified according to its main cellular function. Full definitions/aliases are reported in supplementary appendix S2. \# : significant correlation in the initial analysis. 


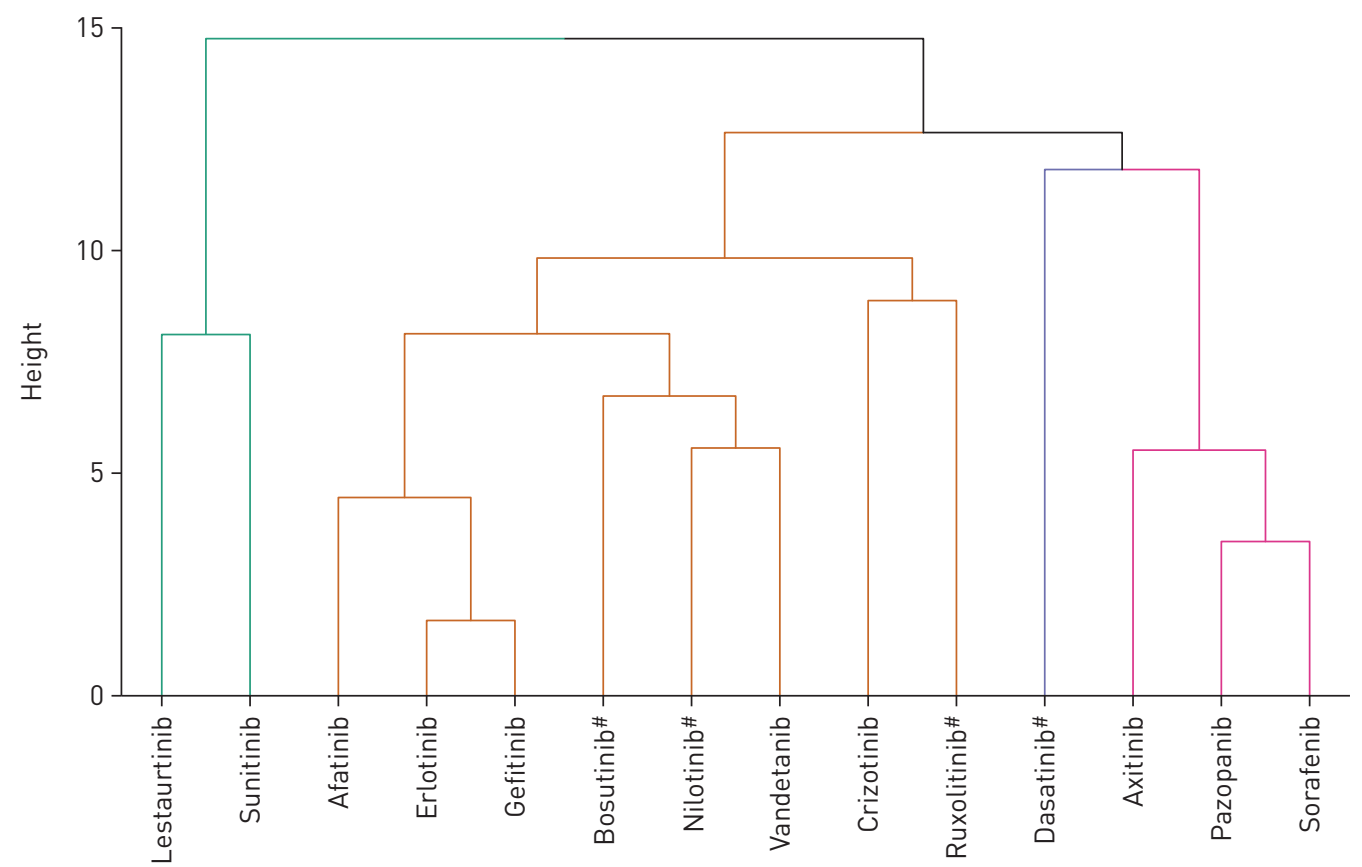

FIGURE 4 Cluster dendrogram of protein kinase inhibitors (PKIs) based on their affinity profile. * : PKI with a significant pharmacovigilance disproportionality signal.

pharmacovigilance database VigiBase at the date of the extraction, 286834 ICSRs were associated the 28 selected PKIs, including 442 PAH cases. Disproportionality analysis showed that dasatinib, bosutinib, ponatinib, ruxolitinib and nilotinib displayed a significant pharmacovigilance signal. Those results are consistent with the literature, with dasatinib being the most widely implicated PKI in induction or aggravation of PAH [5, 20-23]. More recently, bosutinib, ponatinib and ruxolitinib were also linked to PAH $[6,26]$. Results for nilotinib seem less robust because the pharmacovigilance disproportionality signal disappeared in the sensitivity analysis and high dosages were used for a third of the cases. Moreover, well-documented case reports are still lacking in the literature for nilotinib. The pharmacovigilance signal found for ruxolitinib could also be questioned because ruxolitinib is prescribed in the treatment of polycythaemia vera and essential thrombocythaemia, which are recognised causes of pulmonary hypertension. Otherwise, a published case series suggested that lapatinib, a PKI used in breast cancer with human epidermal growth factor receptor mutations, might also cause $\mathrm{PAH}$, but only one of the six

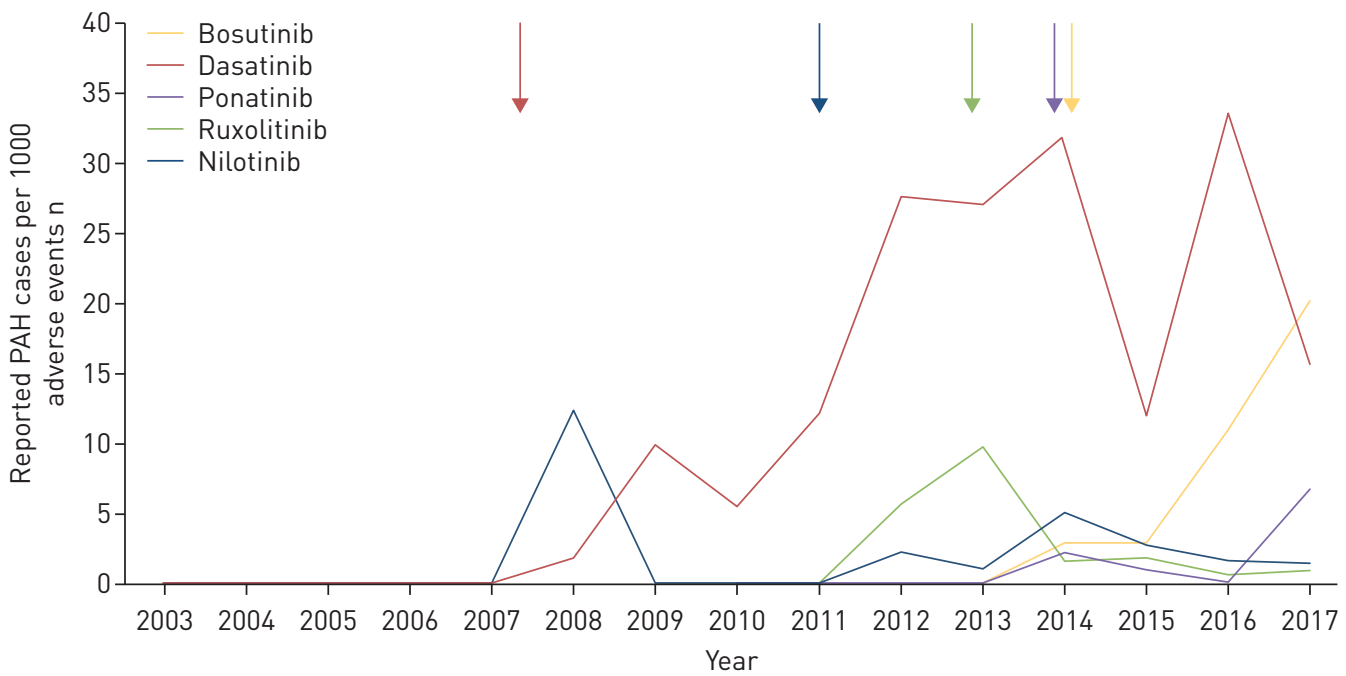

FIGURE 5 Proportion of reported pulmonary arterial hypertension (PAH) cases per 1000 reported adverse events per year for the five protein kinase inhibitors with a significant disproportionality signal. The arrows indicate the first published case reports in MEDLINE for each drug. 
patients presented in this case series had right heart catheterisation confirming pre-capillary PAH [27]. In our study, lapatinib showed a weak, nonsignificant disproportionality signal with a ROR of 1.13 (95\% CI 0.61-2.10). Although not included in our study because of a lack of reported ICSRs in VigiBase, lorlatinib has recently been linked to PAH [7]. Further studies are needed to confirm these first reports.

The correlation analysis showed that c-Src, c-Yes, Lck, Lyn and TEC were highly correlated to PAH reporting risk. The Src tyrosine kinase family contains nine members: three of them (Src, Fyn and Yes) are ubiquitously distributed and six (Blk, Yrk, Fgr, Hck, Lck and Lyn) are variously expressed depending on the tissue. Src tyrosine kinases are crucial for TWIK-related acid sensitive potassium 1 (TASK-1) potassium channel functioning, acting as a cofactor [28]. Mimicking hypoxia conditions, inhibition of Src kinases decreases TASK-1 activity resulting in an intracellular calcium level increase, and thus enhancing vasoconstriction and vascular remodelling [28]. However, these findings have to be balanced by the dasatinib dosage studied, which corresponded to 500 times the clinical dose. Beyond inhibition of such protein kinases, dasatinib might induce apoptosis and endothelial cell dysfunction through an increase of mitochondrial reactive oxygen species (ROS) that is independent from Src family kinase inhibition [29]. However, there are no significant changes in pulmonary haemodynamic parameters in rats treated daily with high doses of dasatinib (10 times the clinical doses) for 4 weeks [29]. Given the probable influence of PKI dosage on the onset of PAH, secondary targets may also have an important contribution that should be further elucidated in pre-clinical research [30-32].

TEC and Lyn have been linked to pleural effusions through an immune-mediated mechanism and could represent a common signalling pathway explaining the high proportion of such disorders in PKI-related PAH cases $[14,33]$. Consistent with the high incidence of dasatinib-induced pleural effusion, rats treated with high doses of dasatinib developed pleural effusion following a period of at least 5 weeks, supporting a direct link between high doses of dasatinib and the development of pleural effusion [34]. Interestingly, this work highlights that high circulating levels of dasatinib alter pulmonary endothelial permeability in a ROS-dependent manner in vitro and in vivo, leading to pleural effusion.

Members of the bone morphogenetic protein signalling pathway showed heterogeneous results in our study. Although ALK1, ALK5 and BMPR1 showed a positive correlation in the main or sensitivity analysis, BMPR2, the primary cause of heritable PAH, did not show any correlation in our study. The bone morphogenetic protein signalling pathway is involved in cell proliferation, mitochondrial dysfunction and inflammation [18]. Mutation of BMPR2, the gene coding for the BMPR2 receptor, accounts for $70-80 \%$ of heritable PAH; furthermore, BMPR2 concentration has also been shown to be reduced in lung tissue from patients with $\mathrm{PAH}$ [35]. However, estimates indicate that only $\sim 20 \%$ of individuals with a known genetic mutation in BMPR2 will develop PAH during their life, thus BMPR2 mutation is required but is not sufficient alone for phenotypic expression and increases an individual's chance of developing $\mathrm{PAH}[18,36]$. Interestingly, CARUso et al. [37] recently showed that BMPR2 reduction, through the microRNA miR-124, leads to the mitochondrial Warburg phenotype and may explain the mitochondrial increased ROS found by GUIGNABERT et al. [29].

The absence of an association between platelet-derived growth factor and vascular endothelial growth factor protein kinases reinforces the fact that vascular remodelling is not a major component of PAH induced by PKIs, which is consistent with the observations of PAH reversal upon PKI discontinuation. Despite this, there is some evidence suggesting that irreversible PAH should occur through ROS generation [26, 38].

Genetic mutations are considered to be permissive of disease and require additional epigenetic, inflammatory or environmental factors for the development of PAH in individuals with those mutations [39]. Similarly, and based on in vitro and in vivo findings, PKIs increase the risk of developing PAH but require a comparable genetic, epigenetic or environmental "second hit", which remains to be identified [29]. According to published case series, a higher proportion of males may develop PKI-induced PAH, while the incidence of $\mathrm{PAH}$ is fourfold higher in females than in males in the general population [18]. It is known that males have a worse prognosis mainly because of a maladaptive response of the right ventricle to $\mathrm{PAH}$; we thus cannot exclude a participation of hormones and sex in triggering PAH [40].

In the cluster analysis, we tried to identify a PKI family specifically involved in PAH. The results are mainly in accordance with the literature and consistent with the chemical structure of PKIs [41]. Interestingly, the dasatinib affinity profile for protein kinases involved in $\mathrm{PAH}$ seems unique among the drug class. However, PKIs such as vandetanib or crizotinib, which share a similar affinity profile to that of bosutinib, nilotinib and ruxolitinib, but which are used in solid-organ malignancies, are not associated with the reporting of PAH (figure 4). This observation may help to elucidate the role of the underlying haematological disease in the genesis of PAH beyond inhibition of protein kinases.

Given that pharmacovigilance notifications are based on a spontaneous reporting system, the number and proportion of cases reported for a medicinal product may vary depending on many factors, such as media 
safety alerts, time since marketing or selective notification. Thus, the exact population exposed to a given drug is unknown. Illustrating this variability, the time-trend analysis showed a large increase in the rate of reporting after the first case series and case report publications. However, despite these biases, a correlation between relative risks and a measure of disproportionality was found [11]. Moreover, while we retrieved all cases for selection in this study we cannot exclude that instances of spurious PAH were included; indeed, only two cases reported abnormal right heart catheterisation results. Unfortunately, the medications introduced after the onset of the adverse event are not fulfilled in the database to avoid spurious pharmacovigilance signals, thus they could not be used for case selection. In two cases (one with dasatinib and one with ruxolitinib) a previous exposure to interferons was found, but the link with PAH onset was not considered strong enough to be excluded. Furthermore, new onset and aggravation of PAH were considered similar. Unfortunately, our study of comedications, associated pathologies and drug dosages was limited by the high rate of missing data in the ICSRs reported in VigiBase. This reinforces the importance of reporting all suspected ADRs on pharmacovigilance systems in order to improve their efficiency [42].

In the present pharmacovigilance-pharmacodynamic analysis, we assumed that PAH was caused by a single protein kinase and we did not account for co-inhibition of multiple protein kinases. However, we tried to address this limitation by performing a cluster analysis to identify at-risk groups of PKIs. Lastly, our study was not able to detect inhibition/activation of non-protein kinase cellular targets (e.g. proteasomes, G protein-coupled receptors, voltage-gated ion channels or ligand-gated ion channels). Therefore, the role of other targets in the pathogenesis of PKI-induced PAH cannot be ruled out.

\section{Conclusions}

To the best of our knowledge, this is the first pharmacovigilance analysis to investigate the risk of PAH associated with PKIs. The disproportionality analysis showed that dasatinib, as well as bosutinib, ponatinib, ruxolitinib and nilotinib, had a significant disproportionality signal. This study highlights potential the roles of Src protein kinases family and TEC in PAH induced by PKIs. Overall, this study contributes to a better understanding of PAH induced by PKIs and to identifying potential targets of interest that need to be further explored.

Conflict of interest: L. Cornet has nothing to disclose. C. Khouri has nothing to disclose. M. Roustit reports grants from United Therapeutics outside the submitted work. C. Guignabert has nothing to disclose. M-C. Chaumais reports nonfinancial support from Bayer and personal fees from Actelion, outside the submitted work. M. Humbert reports personal fees from Actelion, Bayer, GSK, Merck and United Therapeutics, during the conduct of the study. B. Revol has nothing to disclose. F. Despas has nothing to disclose. D. Montani reports grants and personal fees from Actelion and Bayer, and personal fees from BMS, GSK, MSD and Pfizer, outside the submitted work. J-L. Cracowski reports grants from Bioprojet and Topadur, outside the submitted work.

\section{References}

1 Galiè N, Humbert M, Vachiery J-L, et al. 2015 ESC/ERS Guidelines for the diagnosis and treatment of pulmonary hypertension: The Joint Task Force for the Diagnosis and Treatment of Pulmonary Hypertension of the European Society of Cardiology (ESC) and the European Respiratory Society (ERS). Eur Respir J 2015; 46: 903-975.

2 Shimoda LA, Laurie SS. Vascular remodeling in pulmonary hypertension. J Mol Med 2013; 91: 297-309.

3 Humbert M, Morrell NW, Archer SL, et al. Cellular and molecular pathobiology of pulmonary arterial hypertension. J Am Coll Cardiol 2004; 43: S13-S24.

4 Mattei D, Feola M, Orzan F, et al. Reversible dasatinib-induced pulmonary arterial hypertension and right ventricle failure in a previously allografted CML patient. Bone Marrow Transplant 2009; 43: 967-968.

5 Rasheed W, Flaim B, Seymour JF. Reversible severe pulmonary hypertension secondary to dasatinib in a patient with chronic myeloid leukemia. Leuk Res 2009; 33: 861-864.

6 Low AT, Howard L, Harrison C, et al. Pulmonary arterial hypertension exacerbated by ruxolitinib. Haematologica 2015; 100: e244-e245.

7 Chabrol A, Mayenga M, Hamid AM, et al. Lorlatinib-induced pulmonary arterial hypertension. Lung Cancer 2018; 120: 60-61.

8 Hickey PM, Thompson AAR, Charalampopoulos A, et al. Bosutinib therapy resulting in severe deterioration of pre-existing pulmonary arterial hypertension. Eur Respir J 2016; 48: 1514-1516.

9 Bate A, Evans SJW. Quantitative signal detection using spontaneous ADR reporting. Pharmacoepidemiol Drug Saf 2009; 18: 427-436.

10 Candore G, Juhlin $\mathrm{K}$, Manlik $\mathrm{K}$, et al. Comparison of statistical signal detection methods within and across spontaneous reporting databases. Drug Saf 2015; 38: 577-587.

11 Maciá-Martínez M-A, de Abajo FJ, Roberts G, et al. An empirical approach to explore the relationship between measures of disproportionate reporting and relative risks from analytical studies. Drug Saf 2016; 39: 29-43.

12 Raschi E, Poluzzi E, Salvo F, et al. Pharmacovigilance of sodium-glucose co-transporter-2 inhibitors: what a clinician should know on disproportionality analysis of spontaneous reporting systems. Nutr Metab Cardiovasc Dis 2018; 28: 533-542.

13 Khouri C, Lepelley $\mathrm{M}$, Roustit $\mathrm{M}$, et al. Comparative safety of drugs targeting the nitric oxide pathway in pulmonary hypertension: a mixed approach combining a meta-analysis of clinical trials and a disproportionality analysis from the World Health Organization pharmacovigilance database. Chest 2018; 154: 136-147. 
14 Mahé J, de Campaigno EP, Chené A-L, et al. Pleural adverse drugs reactions and protein kinase inhibitors: identification of suspicious targets by disproportionality analysis from VigiBase: pleural adverse drugs reactions and protein kinase inhibitors. Br J Clin Pharmacol 2018; 84: 2373-2383.

15 Orcholski ME, Yuan K, Rajasingh C, et al. Drug-induced pulmonary arterial hypertension: a primer for clinicians and scientists. Am J Physiol Lung Cell Mol Physiol 2018; 314: L967-L983.

16 Patras de Campaigno E, Bondon-Guitton E, Laurent G, et al. Identification of cellular targets involved in cardiac failure caused by PKI in oncology: an approach combining pharmacovigilance and pharmacodynamics. $\mathrm{Br} \mathrm{J} C l i n$ Pharmacol 2017; 83: 1544-1555.

17 Frost AE, Barst RJ, Hoeper MM, et al. Long-term safety and efficacy of imatinib in pulmonary arterial hypertension. J Heart Lung Transplant 2015; 34: 1366-1375.

18 Thenappan T, Ormiston ML, Ryan JJ, et al. Pulmonary arterial hypertension: pathogenesis and clinical management. BMJ 2018; 360: j5492.

19 Harding SD, Sharman JL, Faccenda E, et al. The IUPHAR/BPS Guide to PHARMACOLOGY in 2018: updates and expansion to encompass the new guide to IMMUNOPHARMACOLOGY. Nucleic Acids Res 2018; 46: D1091-D1106.

20 van Puijenbroek EP, Bate A, Leufkens HGM, et al. A comparison of measures of disproportionality for signal detection in spontaneous reporting systems for adverse drug reactions. Pharmacoepidemiol Drug Saf 2002; 11: 3-10.

21 EudraVigilance Expert Working Group. Guideline on the Use of Statistical Signal Detection Methods in the EudraVigilance Data Analysis System (EMEA/106464/2006 rev.1). 2008. www.ema.europa.eu/docs/en_GB/ document_library/Regulatory_and_procedural_guideline/2009/11/WC500011434.pdf Date last accessed: April 27, 2017.

22 Khouri C, Cracowski J-L, Roustit M. SGLT-2 inhibitors and the risk of lower-limb amputation: is this a class effect? Diabetes Obes Metab 2018; 20: 1531-1534.

23 Bland JM, Altman DG. Multiple significance tests: the Bonferroni method. BMJ 1995; $310: 170$.

24 Davis MI, Hunt JP, Herrgard S, et al. Comprehensive analysis of kinase inhibitor selectivity. Nat Biotechnol 2011; 29: 1046-1051.

25 Kassambara A. Practical Guide to Cluster Analysis in R: Unsupervised Machine Learning. Scotts Valley, CreateSpace, 2017.

26 Weatherald J, Chaumais M-C, Montani D. Pulmonary arterial hypertension induced by tyrosine kinase inhibitors. Curr Opin Pulm Med 2017; 23: 392-397.

27 Alkhatib Y, Albashaireh D, Al-Aqtash T, et al. The role of tyrosine kinase inhibitor "Lapatinib" in pulmonary hypertension. Pulm Pharmacol Ther 2016; 37: 81-84.

28 Nagaraj C, Tang B, Balint Z, et al. Src tyrosine kinase is crucial for potassium channel function in human pulmonary arteries. Eur Respir J 2013; 41: 85-95.

29 Guignabert C, Phan C, Seferian A, et al. Dasatinib induces lung vascular toxicity and predisposes to pulmonary hypertension. J Clin Invest 2016; 126: 3207-3218.

30 Bonnet S, Provencher S, Guignabert C, et al. Translating research into improved patient care in pulmonary arterial hypertension. Am J Respir Crit Care Med 2017; 195: 583-595.

31 Humbert M, Guignabert C, Bonnet S, et al. Pathology and pathobiology of pulmonary hypertension: state of the art and research perspectives. Eur Respir J 2019; 53: 1801887.

32 Bonniaud P, Fabre A, Frossard N, et al. Optimising experimental research in respiratory diseases: an ERS statement. Eur Respir J 2018; 51: 1702133

33 Kelly K, Swords R, Mahalingam D, et al. Serosal inflammation (pleural and pericardial effusions) related to tyrosine kinase inhibitors. Target Oncol 2009; 4: 99-105.

34 Phan C, Jutant E-M, Tu L, et al. Dasatinib increases endothelial permeability leading to pleural effusion. Eur Respir J 2018; 51: 1701096.

35 Atkinson C, Stewart S, Upton PD, et al. Primary pulmonary hypertension is associated with reduced pulmonary vascular expression of type II bone morphogenetic protein receptor. Circulation 2002; 105: 1672-1678.

36 Austin ED, Loyd JE. Genetics and mediators in pulmonary arterial hypertension. Clin Chest Med 2007 ; 28 : 43-57.

37 Caruso P, Dunmore BJ, Schlosser K, et al. Identification of miR-124 as a major regulator of enhanced endothelial cell glycolysis in pulmonary arterial hypertension via PTBP1 and PKM2. Circulation 2017; 126: 2451-2467

38 Daccord C, Letovanec I, Yerly P, et al. First histopathological evidence of irreversible pulmonary vascular disease in dasatinib-induced pulmonary arterial hypertension. Eur Respir J 2018; 51: 1701694

39 Pousada G, Baloira A, Valverde D. Complex inheritance in pulmonary arterial hypertension patients with several mutations. Sci Rep 2016; 6: 33570.

40 Shapiro S, Traiger GL, Turner M, et al. Sex differences in the diagnosis, treatment, and outcome of patients with pulmonary arterial hypertension enrolled in the registry to evaluate early and long-term pulmonary arterial hypertension disease management. Chest 2012; 141: 363-373.

41 Green MR, Newton MD, Fancher KM. Off-target effects of BCR-ABL and JAK2 Inhibitors. Am J Clin Oncol 2016 39: 76-84.

42 Chaumais M-C, O'Connell C, Savale L, et al. Pharmacovigilance in a rare disease: example of the VIGIAPATH program in pulmonary arterial hypertension. Int J Clin Pharm 2018; 40: 790-794. 